\section{SPEER, Heino}

Ehem. Forschungsstellenleiter

Heidelberger Akademie der Wissenschaften
DOI: 10.15170/DIKE.2020.04.01.16.

\title{
István [Stefan von] Werbőczy: Digitale Annäherungen an einen großen ungarischen Juristen
}

\author{
Ein Arbeitsbericht ${ }^{1}$
}

\section{Persönliches}

Es begann ein paar Jahre vor dem Datum meiner Pensionierung. Mir wurde bewusst, dass ich im Ruhestand alle wissenschaftlichen Ressourcen des Deutschen Rechtswörterbuchs ${ }^{2}$ (das ich 34 Jahre die Ehre hatte zu leiten) nicht mehr zur Verfügung haben würde. Insbesondere die deutschsprachigen juristischen Drucke des 16. Jahrhunderts, die mich damals besonders interessierten, würden nur mehr mit Schwierigkeiten für mich erreichbar sein. Es wurde mir aber auch bewußt, dass ich aus meiner privilegierten Stellung eines Wissenschaftlers mit einer ausgezeichneten Institutsbibliothek und einer noch besseren Universitätsbibliothek in einen Normalzustand fallen würde, den ich mit allen institutionell nicht oder kaum eingebundenen Wissenschaftlern und Wissenschaftlerinnen teilen würde. Mir schien, wie auch schon bei der Digitalisierung des Rechtswörterbuchs, durch das Internet eine Möglichkeit gegeben zu sein, nur lokal vorliegende Ressourcen weltweit verfügbar zu machen und damit eine „Demokratisierung des Wissens" zu erreichen. Noch war es genug Zeit, um bei der Deutschen Forschungsgemeinschaft gemeinsam mit dem Max-Planck-Institut für Europäische Rechtsgeschichte ${ }^{3}$ und der damaligen Professur für Historisch-Kulturwissenschaftliche Informationsverarbeitung der Universität zu Köln ${ }^{4}$ (Prof. Dr. Manfred Thaller) einen Projektantrag einzureichen, dessen Ziel es war, in einer virtuellen Bibliothek alle deutschsprachigen juristischen Erstdrucke vom Beginn des Buchdrucks bis 1600 zusammenzuführen - eine Bibliothek, wie sie realiter nirgendwo vorhanden war.

Der Antrag wurde genehmigt und nun stehen in dem Projekt DRQEdit 560 Drucke als Faksimile und teilweise als Volltext unentgeltlich im Internet zur Verfügung. Die langjährige Arbeit mit historischen Quellen von der Völkerwanderungszeit bis etwa 1820 (ca. 8.000 Siglen des DRW $^{6}$ erschließen diese Quellen) erleichterte mir die Vorbereitung des Projektantrags, aber dabei stieß ich doch auf einige Titel, die mir bislang unbekannt waren. Dazu gehörte auch eine deutsche Übersetzung des Opus tripartitum von Istvan Werböcsyy (vgl. die Titelaufnahme in DRQEdit). Nicht

\footnotetext{
1 Eine HTML-Version dieses Arbeitsberichtes mit anklickbaren Links ist hier zu finden: http://repertorium.at/sl/arbeitsbericht_2020.html. (Alle Internetseiten sind zuletzt am 09. 06. 2020 aufgerufen worden.)

${ }^{2}$ https://drw-www.adw.uni-heidelberg.de/drw-cgi/zeige.

3 https://www.rg.mpg.de/.

${ }^{4}$ https://dh.phil-fak.uni-koeln.de/hki.

${ }^{5}$ https://drw-www.adw.uni-heidelberg.de/drqedit-cgi/zeige.

${ }^{6}$ https://drw-www.adw.uni-heidelberg.de/drw-cgi/zeige?index=siglen.
} 
alle Ziele konnten innerhalb des verfügbaren Zeitrahmens erreicht werden, so dass die Gutachter der DFG eine Weiterführung in einer zweiten Projektphase empfohlen hatten. Dazu kam es jedoch nicht, da ich 2010 auf Grund familiärer Umstände meinen Zweitwohnsitz nach Klagenfurt in Kärnten verlegte und die räumliche Entfernung trotz aller Möglichkeiten des Internet mir persönlich eine weitere Betreuung des Projektes unmöglich zu machen schien. Zu den offenen Werken gehörte auch das Opus Tripartitum, das zwar als Digitalisat eingebunden, aber nicht weiter bearbeitet worden war. Denn das erklärte Ziel von DRQEdit war ja die Herstellung eines maschinenlesbaren Volltextes all der in ihm versammelten Druckwerke gewesen.

Im Lauf der folgenden Jahre baute ich ein privates Webportal mit dem Titel Repertorium digitaler Quellen zur österreichischen und deutschen Rechtsgeschichte in der Früben Neuzeit (im Weiteren: Repertorium) auf. Da dieses Portal in keine Institution eingebunden war und die - marginale Finanzierung nur von mir getragen wurde, konnte ich meinen persönlichen Interessen und den Zufälligkeiten meiner Entdeckungen im digitalen Raum folgen. Hierzu gehörte beispielsweise die Konfessionsgeschichte der Protestanten in Österreich mit den dazugehörigen Quellen. ${ }^{8}$ Gegenwärtig sind in diesem Repertorium 182 rechtshistorische Quellenwerke und 180 Werke, die der Sekundärliteratur angehören, versammelt. Dabei verdanke ich es dem Entgegenkommen einiger bedeutender Rechtshistoriker (zum Teil auch dem ihrer Erben), dass ich auch Werke der Sekundärliteratur digitalisieren konnte, die noch nicht gemeinfrei waren. Ich darf hier nur stellvertretend die Namen Hermann Baltl, Wilhelm Brauneder, Helmut Coing und Gunter Wesener nennen.

Ursprünglich war es meine Absicht, mich auf österreichische Quellen zu beschränken aber mir wurde sehr bald wieder bewußt, dass dies ein völlig unhistorischer Zugang zu einem Bereich sein würde, der weder mit dem heutigen staatsrechtlichen Begriff „Österreich” noch auch mit „Altösterreich” oder „Erblande” zu fassen war. Da mein Ausgangspunkt und mein ursprüngliches Interesse immer ein irgendwie gearteter österreichischer oder habsburgischer Bezug dieser Werke war, finden sich im Webportal beispielsweise Digitalisate des Görzer Statutbuchs (um 1500), ${ }^{9}$ der Clesischen Statuten von 1528, ${ }^{10}$ des Meraner Stadtrechts (nach 1337), ${ }^{11}$ des Freiburger Stadtrechts von Ulrich Zasius (1520) ${ }^{12}$ sowie der Übersetzung des Eigenlandrechts der Siebenbürger Sachsen von 1583. ${ }^{13}$ Und schließlich eine rein ungarische Quelle wie Werböcayys Opus Tripartitum in ihrer frühneuzeitlichen deutschen Übersetzung.

\section{Annäherungen an die deutsche Übersetzung}

Man wird es einem deutschen Wissenschaftler, von dem nicht so genau auszumachen ist, ob er nun eigentlich Lexikograph oder Rechtshistoriker ist, nachsehen, wenn es bei seiner Begegnung mit Werböcay zunächst nur um eine Annäherung gehen konnte. Immerhin war der erste Begegnungspunkt interessant genug: Was brachte einen deutschen Juristen und einen Wiener

\footnotetext{
${ }^{7}$ http:// repertorium.at.

8 http://repertorium.at/protestantismus.html.

${ }^{9}$ http://repertorium.at/qu/goerzstat.html.

${ }^{10} \mathrm{http}: / /$ www.stabat.it $/ \mathrm{sites} / \mathrm{all} / \mathrm{modules} /$ digitalib $/$ index.php?dirname $=243 \&$ fnum $=266 \&$ fui $=\mathrm{embed} \# \mathrm{page} / 1 / \mathrm{mod}$ e/2up.

11 http://repertorium.at/qu/1338_meranstr.html.

12 http://repertorium.at/qu/1520_freiburgstr.html.

13 https://drw-www.adw.uni-heidelberg.de/drqedit-cgi/zeige? sigle=siebblr. +1583 .
} 
Drucker dazu, mit einer sicherlich nicht unerheblichen Anstrengung eine deutsche Übersetzung eines ungarischen, lateinisch geschriebenen „Gesetzbuches”, das nie eine staatliche Inkraftsetzung erfahren hatte, anzufertigen?

Die deutsche Übersetzung von Werböcsy ist 1599 in Wien in der Buchdruckerei Formica gedruckt worden. Der Übersetzer gibt sich als „AVGVSTINVS VVAGNERUS Villseccensis, I.V. Candidatus” zu erkennen und widmet sein Werk „Denen Edlen / Ehrnvesten / Fürsichtigen vnd Wolweisen Herrn N. Richtern / Burgermeistern vnnd Rath der Königklichen freyen HaubtStatt Preßburg in Hungern" [Vorrede]. ${ }^{14}$ Als Begründung seines Vorhabens gibt er an: es sei ,angesprochen worden / dem gemeinen Mann / und den jenigen / die der Lateinischen vnd Hungerischen Sprachen nicht erfahren sind / zu gutem solche meine einfaltige Translation in offentlichen Druck verfertigen zu lassen". Und immerhin fand diese Übersetzung die obrigkeitliche Billigung: ,entlichen auff begerten vñ erfolgten gnugsamen bericht / vnd fleissige Reuision beeder hocblöblichen Regierung und Vniuersitet der auch bocblöbliche [n] Herrn Hungrischen Räth guetachten / auffjüngst verwichenen vñ allbier zu Preßburg dieses Neunvndneunzigisten Jabrs gehaltenen Landtag darüber genedigist erfordert / vnd weiln auch wolgedachte Herrn Räth nicht darwider gewesen / als haben jhr Fürst: Durchl: solche Publication, beneben auch ein Prinilegium auff acht Jabrlang nicht nach zudrucken / in gedachtem Hungerischen Landtag mir genedigst vervilligt." Über die Person von Augustin Wagner war wenig zu erfahren: Er ist zwar in der sog. Gemeinsamen Normdate ${ }^{15}$ der Deutschen Nationalbibliothek verzeichnet [Datensatz], ", aber der Vermerk „Respondent an der juristischen Fakultät der Universität Jena” und das Tätigkeitsjahr 1592 führten in dem Fall auch nicht weiter. Eine Anfrage an das Archiv der Universität Jena verlief im Sande.

Aber es ging mir ja letztlich nur darum, einen maschinenlesbaren Text dieses Druckes herzustellen, ganz gleich, wer dessen Urheber bzw. Übersetzer war. Der in DRQEdit verlinkte Druck der Staatsbibliothek Berlin ${ }^{17}$ stand als Quelle bereits zur Verfügung. Seit dieser Digitalisierung waren bereits einige Jahre vergangen und es hat sich in solchen Fällen immer als sinnvoll erwiesen, weitere und neuere Digitalisate dieses Druckes zu suchen. Denn der technische Fortschritt könnte es mit sich bringen, dass bei einem neueren Digitalisat Informationen hinzukommen, die für eine Weiterbearbeitung wichtig sein können. Für solche Fälle bietet der Karlsruher Virtuelle Katalog ${ }^{18}$ gute Recherchemöglichkeiten, bei denen man beispielsweise als Filter das Vorliegen als digitales Medium wählen kann. Diese Recherchen im KVK führten zu zwei weiteren Digitalisaten: Einmal handelt es sich um ein Google-Digitalisat ${ }^{19}$ eines Exemplares der British Library. Ein zweites Exemplar wurde im Rahmen der Digitalisierungskampagne der Bayerischen Staatsbibliothek digitalisiert. ${ }^{20}$ Diese drei Digitalisate waren meine einzige Arbeitsgrundlage.

Im Wesentlichen gibt es zwei Methoden, um ein Druckwerk maschinenlesbar zu machen. Die erste besteht darin, Seite für Seite und Zeile für Zeile in eine Computerdatei schreiben zu lassen. Während des Projektverlaufs von DRQEdit wäre eine dieser Faksimiledateien nach Vietnam

\footnotetext{
14 http://repertorium.at/qu/1599_opus_tripartitum_dt.html\#Vorrede_Wagner.

15https://www.dnb.de/DE/Professionell/professionell_node.html;jsessionid=B7E4678FB4C8285C72E18544E902 1FCF.internet561\#sprg315806.

16 https://portal.dnb.de/opac.htm?method=simpleSearch\&cqlMode=true\&query =nid\%3D132379341.

17 https://digital.staatsbibliothek-berlin.de/werkansicht/?PPN=PPN626373948.

18 http:/ / kvk.bibliothek.kit.edu/?digitalOnly $=0$ \&embedFulltitle $=0 \& n e w T a b=0$.

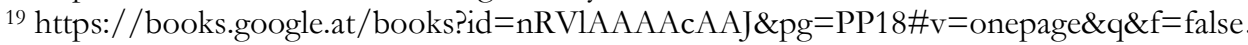

20 https:/ / reader.digitale-sammlungen.de//resolve/display/bsb10152166.html.
} 
geschickt worden, um dort nach vorgegebenen Regeln abgeschrieben zu werden, wobei ich seinerzeit gelernt hatte, dass es besser ist, die Abschrift von Personen durchführen zu lassen, die die fragliche Sprache nicht kennen: So wird man vor faux amis ${ }^{21}$ bewahrt. Die andere Möglichkeit wäre es, ein auf die Erkennung von Druckzeichen spezialisiertes Computerprogramm [OCR $=$ Optical Character Recognition $]^{22}$ mit den Einzelseiten zu füttern und zu schauen, in welcher Präzision die Druckzeichen in Computerschrift umgesetzt worden sind. Bei moderneren Antiquadrucken funktioniert dies ganz gut, da diese Schriftfonts unter dem Gesichtspunkt der guten Lesbarkeit entwickelt worden sind. Sieht man sich aber frühere Schriften an, so wird man auch damit konfrontiert, dass diese Druckschriften für ungeübte Leser teilweise schwer zu lesen sind. Zu viele Buchstaben sind sich ähnlich, etwa das Schaft- „, $\mathrm{s}^{\prime \prime}$ und das kleine „f“, das kleine „, c“ und „e" usw. In manchen dieser Programme ist eine Lern- bzw. Trainingsfunktion eingebaut, mit der man Buchstaben, die das Programm nicht richtig zuordnen kann, für dieses und die weiteren Male mit einer eindeutigen Zuordnung versieht. Gegenwärtig scheinen die großen Bibliotheken bei der Digitalisierung auch älterer Quellen OCR-Programme mitlaufen zu lassen, die teilweise recht brauchbare Ergebnisse liefern. Für die eben genannte Einschränkung hinsichtlich der Brauchbarkeit will ich ein beliebiges Beispiel zeigen:

\begin{tabular}{|c|c|}
\hline & Kor \\
\hline 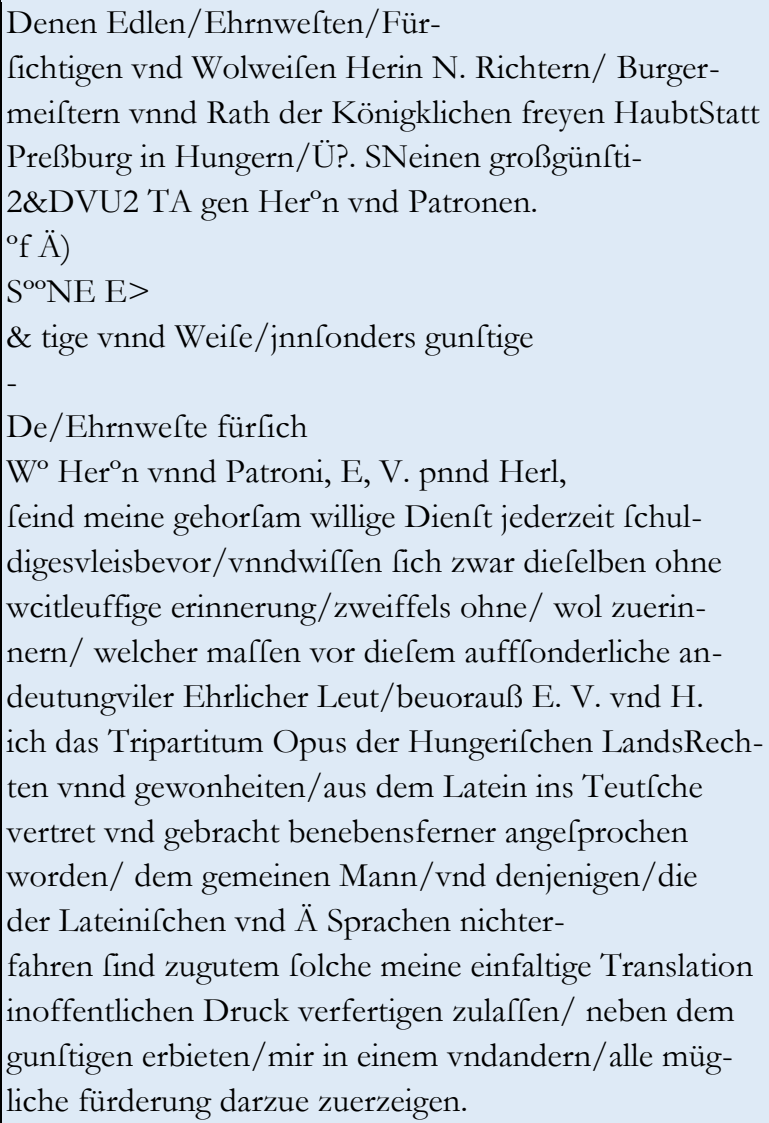 & $\begin{array}{l}\text { Denen Edlen / Ehrnveften / Für= } \\
\text { fichtigen vnd Wolweifen Herrn N. Richtern / Burger= } \\
\text { meiftern vnnd Rath der Königklichen freyen HaubtStatt } \\
\text { Preßburg in Hungern / \&c. Meinen großgünfti= } \\
\text { gen Herrn vnd Patronen. } \\
\text { EDle / Ehrnvefte / fürfich= } \\
\text { tige vnnd Weife / jnnfonders gunftige } \\
\text { Herrn vnnd Patroni, E.V. vnnd Herrl. } \\
\text { feind meine gehorfam willige Dienft jederzeit schul= } \\
\text { diges vleis bevor / vnnd wiffen fich zwar diefelbe ohne } \\
\text { weitleuffige erinnerung / zweiffels ohne / wol zuerin= } \\
\text { nern / welcher maffen vor diefem auff fonderliche an= } \\
\text { deutung viler Ehrlicher Leut / beuor auß E.V. vnd H. } \\
\text { ich das Tripartitum Opus der Hungerischen LandsRech= } \\
\text { ten vnnd gewonheiten / aus dem Latein ins Teutfche } \\
\text { vertiret vnd gebracht : benebens ferner angefprochen } \\
\text { worden / dem gemeinen Mann / vnd den jenigen / die } \\
\text { der Lateinifchen vnd Hungerifchen Sprachen nicht er= } \\
\text { fahren sind / zu gutem folche meine einfaltige } \\
\text { Translation } \\
\text { in offentlichen Druck verfertigen zu laffen / neben dem } \\
\text { gunftigen erbieten / mir in einem vnd andern / alle } \\
\text { müg= } \\
\text { liche fürderung darzue zu erzeigen. }\end{array}$ \\
\hline
\end{tabular}

Quelle: Digitalisat der Bayerischen Staatsbibliothek des dortigen Exemplars der Übersetzung von Augustin Wagner23

\footnotetext{
${ }^{21}$ https://de.wikipedia.org/wiki/Falscher_Freund.

22 https://de.wikipedia.org/wiki/Texterkennung.

${ }^{23}$ https:// reader.digitale-sammlungen.de// resolve/display/bsb10152166.html.
} 
Die Ergebnisse einer maschinellen Zeichenerkennung werden gerade für den Bereich der frühneuzeitlichen Drucke immer einer gründlichen Korrektur bedürfen. Freilich kann diese in mehreren Schritten erfolgen, denn viele Erkennungsfehler sind in gewisser Weise für diesen einen Druck typisch und können daher bei genügender Kenntnis des Textes semiautomatisch bereinigt werden. Dazu muss man Regeln für das Auftreten bestimmter Zeichen herausfinden, aber dann kann man beispielsweise jedes „s" nach einem Leerzeichen (also am Wortbeginn) in ein Schaft-,,s” umwandeln. Oder man wandelt überhaupt jedes kleine „," zunächst in ein Schaft-,,s" um und definiert dann die Stelle, an denen dies nicht passt, etwa am Wortende. Nur muss man dann eben berücksichtigen, dass das Wortende nicht unbedingt nur ein darauf folgendes Leerzeichen voraussetzt, sondern dass alle möglichen Satzzeichen, die unmittelbar auf das „s” folgen, die gleiche Wirkung haben. Und, last, but not least, muss man sich überzeugen, dass in der endgültigen Darstellung, die man gewählt hat, das Schaft-,,s” überhaupt von den Browsern dargestellt werden kann. Was der Texteditor an Zeichen zu verarbeiten vermag, muss in einer HTML-Datei vorsichtshalber als Unicode-Zeichen kodiert werden: „\&\#383;”.

Der OCR-Rohtext musste natürlich strukturiert und die Einteilungen des Werkes mussten in den Text eingetragen werden (Textteil, Buch, Titel, Absatz). Wie es heute state of the art ist, habe ich die Rohdaten in einer XML-Datei bearbeitet und diese entsprechend den Richtlinien der Text Encoding Initiative (TEI Leitseite ${ }^{24}$ markiert. Die XML-Datei wurde dann mit dem OxygenEditor $^{25}$ weiterverarbeitet, bis schließlich als Ergebnis die HTML-Datei im Netz stand.

Der digitalisierte Druck der deutschen Übersetzung des Opus Tripartitum umfasst 402 Images, inklusive des Bucheinbandes und der unbedruckten Seiten. Der Text besteht aus ungezählten und gezählten Seiten, wobei die Zählung eine Blattzählung ist, also zB. $13^{\mathrm{r}}$ und $13^{\mathrm{v}}$ :

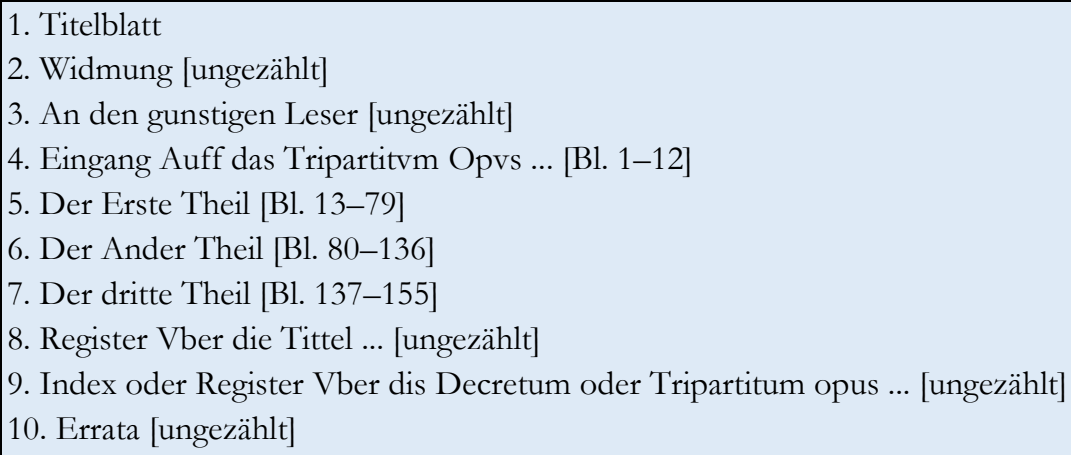

Die Teile 8-10 sind Zusätze gegenüber dem Erstdruck von 1517. Die Herstellung eines brauchbaren maschinenlesbaren Textes war eine zeitaufwendige Angelegenheit und hat sich etwa über ein Jahr erstreckt, ohne dass dies natürlich meine einzige Aufgabe in dieser Zeit gewesen wäre. Vor der Publizierung des Textes in meinem Webportal mussten allerdings noch strukturelle Probleme gelöst werden. Denn der Text sollte schließlich mit den Faksimiles des Druckes verbunden werden, so dass jeder Benutzer / jede Benutzerin die Möglichkeit haben sollte, die Genauigkeit der Transkription zu überprüfen. Es musste also eine Verbindung von jedem

\footnotetext{
24 https:// tei-c.org/.

25 https://www.oxygenxml.com/xml_editor.html.
} 
Abschnitt zu der physischen Seite, auf der er beginnt, geschaffen werden. Genau diese Verknüpfungsmöglichkeit ist der Mehrwert, den das Internet den Geisteswissenschaften (und nicht nur diesen) vermittelt hat. Inzwischen ist eine Unzahl von Anwendungsmöglichkeiten der Internettechniken für die Geisteswissenschaften entwickelt worden, man findet sie unter dem Stichwort „Digital Humanities”.

Für meine konkrete Arbeit an diesem Text bedeutete dies, dass ich ein Referenzexemplar des Druckes festlegen, dieses gegebenenfalls strukturell umarbeiten und dann die Verbindungen über eine Verlinkung jedes Abschnitts mit der entsprechenden Seite vornehmen musste. Als Referenzexemplar wählte ich das Digitalisat der Staatlichen Bibliothek Regensburg [Katalogisat], ${ }^{26}$ weil das Werk als PDF-Datei herunterladbar ${ }^{27}$ und damit lokal bearbeitbar war. Die Herstellung einer lokalen Version auf meinem Rechner wäre zwar nicht unbedingt nötig gewesen, sollte mich aber vor den möglichen Inkonsistenzen einer Internetverbindung bewahren. Die PDF-Datei habe ich - mehrstufig - so weit umgewandelt, dass am Schluss alle Bilddateien in einer Webseite verfügbar waren und dort seitenweise aufgerufen werden können [Seite im Repertorium]. ${ }^{28}$ Dies hat auch den Vorteil, dass alle Bilddateien als sogenannte thumbnails in einer Seitenleiste angezeigt werden, so dass man hierüber relativ leicht zu der interessierenden Seite gelangen kann, ohne eine Bilddatei nach der anderen durchblättern zu müssen.

Auf diese Weise - Auswahl des Exemplares, Bearbeiten eines vorgegebenen OcR-Textes, Markieren des Textes mit Layoutbefehlen, Verlinkung mit den Imagedateien und schließlich dem Herstellen einer HTML-Datei - kam eine digitale Version der deutschen Übersetzung des Opus Tripartitum zustande und ist nun im Internet verfügbar.

\section{Vernetzung mit dem lateinischen Text}

Damit hätte ich die Beschäftigung mit Stefan von Werböcsyy abschließen können. DRQEdit hatte einen weiteren deutschen Text zu integrieren, für die deutschsprachigen Rechtshistoriker gab es einen neuen Zugang zu einem der wichtigsten Werke der ungarischen Rechtsgeschichte, und ich hätte abwarten können, wer davon überhaupt Notiz nehmen würde.

Aber dieser Text stand etwas vereinsamt und herausgelöst aus der gesamten Textgeschichte in meinem Repertorium. Das Mindeste war die Vernetzung zumindest mit dem Originaldruck von 1517. Dann gab es noch diverse Ausgaben im Verlauf der Jahrhunderte, um die ich mich nicht kümmern wollte. Aber es gab auch kanonische Ausgaben im Corpus Juris Hungarici, die erste in der Ausgabe von 1822, ${ }^{29}$ die zweite dann in der Millenniumsedition von $1897 .{ }^{30}$ Wenn ich die deutsche Übersetzung an den lateinischen Urtext binden wollte, wäre wohl der Erstdruck 1517 die richtige Adresse gewesen. Andererseits könnte man bei den beiden Ausgaben aus dem 19. Jahrhundert mit Korrekturen und Verbesserungen rechnen. Leider standen mir keine Entscheidungshilfen zur Verfügung: Die Editionsprinzipien waren natürlich in ungarischer

\footnotetext{
${ }^{26}$ https://www.regensburger-

katalog.de/TouchPoint/perma.do?q $=+1035 \% 3 \mathrm{D} \% 22 \mathrm{BV} 011641894 \% 22+\mathrm{IN}+\% 5 \mathrm{~B} 2 \% 5 \mathrm{D} \& \mathrm{v}=\mathrm{ubrsbr} \& \mathrm{l}=\mathrm{de}$.

27 https://download.digitale-sammlungen.de/BOOKS/download.pl?id=bsb11057202.

28 http://repertorium.at/qu/1599_opus_tripartitum_dt.html.

${ }^{29}$ https://digital.onb.ac.at/OnbViewer/viewer.faces?doc=ABO_\%2BZ15940550X.

${ }^{30}$ https://adtplus.arcanum.hu/hu/view/Eltekonyvek_BE_4107_02/?pg=75\&layout=s.
} 
Sprache verfasst. Daher versuchte ich, probeweise beide Editionen mit einem OcR-Programm zu bearbeiten. Allerdings ergab auch dies keine Entscheidungshilfe, denn bald stellte sich heraus, dass beide Editionen sich zumindest in Kleinigkeiten voneinander unterschieden. Es war und ist mir nicht möglich, im Rahmen meines Projektes so weit in die Geschichte eines solchen Textes zu gehen, dass ich selbständig eine Auswahl würde treffen können. Nach längeren Versuchen blieb eigentlich nur übrig, den Erstdruck als Referenz zu benutzen.

Wenn man einen Blick auf diesen Druck ${ }^{31}$ wirft, wird einem sofort deutlich, dass die automatische Herstellung eines maschinenlesbaren Textes nicht möglich sein würde. Die Edition 1822 ist durch die Österreichische Nationalbibliothek digitalisiert worden und mit etwas Suchen findet man auch den Menüpunkt, durch den das Herunterladen des kompletten Buches möglich ist. Man erhält eine PDF-Datei in der Größe von $881 \mathrm{MB}$, die man entsprechend den eigenen Bedürfnissen nutzen kann. Da es mir um eine Textbasis für eine Transkription ging, musste ich die PDF-Datei in reinen Text umwandeln. Während dies früher nur durch ein OCR-Programm möglich war, bieten heute auch PDF-Editoren eine Funktion an, mit der der Textinhalt in verschiedene Formate exportiert werden kann. Da nun schon ein OCR-Ergebnis vorlag, lag es nahe, diesen Text als Grundlage für eine Transkription des Originaltextes zu benutzen.

Es kam nun darauf an, den ,normalisierten” Text in die Schreibweise des Urtextes zurückzuverwandeln. Dieser Text wies natürlich die üblichen Abkürzungen der damaligen Drucke auf, die das Lesen heute sehr erschweren, wenn man das nicht einmal gelernt hat. In der Ausgabe 1822 waren die Abkürzungen aufgelöst, und dies sollte auch in der Transkription des Druckes 1517 erfolgen. Allerdings stimmte diese Ausgabe nicht diplomatisch genau mit dem Erstdruck überein, so dass ein gründliches Korrekturlesen erforderlich wurde. Als Ergebnis wird aber (sub conditione Jacobaea) erstmals eine diplomatisch genaue Transkription des Erstdruckes zur Verfügung stehen. Wenn auch über den etwas komplizierten Umweg der Digitalisierung der Edition 1822 und die Aufhebung der dort vorgenommenen Normalisierungen.

Es dürfte deutlich geworden sein, dass eine inhaltliche Auseinandersetzung mit diesem Text oder auch nur die Überlegung, ob der Text grammatikalisch korrekt formuliert war, außerhalb meines Interesses lag. Mir kam es nur darauf an, in meinem Repertorium das Handwerkszeug bereitzustellen, damit andere Wissenschaftler und Wissenschaftlerinnen sich ohne Beschränkungen durch die mangelnde Verfügbarkeit der diversen Texte mit ihnen würden beschäftigen können.

Das Ergebnis sieht so aus [Pars 1 Tit. 30 \$3]:

\begin{tabular}{|c|c|c|}
\hline OCR-'T & Korrektur 1822 & Erstdruck 1517 \\
\hline $\begin{array}{l}\text { S. 5. Nam hujus publicatio, } \\
\text { juxta veterem, et } \\
\text { approbatam Regni legem, } \\
\text { non in curia Regiae } \\
\text { Majestatis, sed in faciebus } \\
\text { ipsorum jurium } \\
\text { possessionariorum, maxi me } \\
\text { vero in loco solitae } \\
\text { residentiae illius, qui in }\end{array}$ & $\begin{array}{l}\text { 3. } 3 \text {. Nam hujus publicatio, } \\
\text { juxta veterem, et } \\
\text { approbatam Regni legem, } \\
\text { non in curia Regiae } \\
\text { Majestatis, sed in faciebus } \\
\text { ipsorum jurium } \\
\text { possessionariorum, maxime } \\
\text { vero in loco solitae } \\
\text { residentiae illius, qui in }\end{array}$ & $\begin{array}{l}\text { Nam hui } \\
\text { us publicatio, iuxta ueterem, \& approbatam regni legem / } \\
\text { non in cu } \\
\text { ria regiae maieftatis / fed in faciebus ipforum iurium } \\
\text { poffefsionari= } \\
\text { orum / maxime uero in loco folitae refidentiae illius qui } \\
\text { in femine } \\
\text { defecit / tempore uidelicet legitimae ftatutionis eorum / } \\
\text { praefentibus }\end{array}$ \\
\hline
\end{tabular}

${ }^{31}$ http://repertorium.at/img/img_1517_tripartitum_oenb/original/s003.html. 


\begin{tabular}{|c|c|c|}
\hline $\begin{array}{l}\text { semine defecit, tempore } \\
\text { videlicet legitimae statulionis } \\
\text { eoruin, praescntibus vicinis, } \\
\text { et commetaneis, fieri debet, } \\
\text { et a die ejuscemodi } \\
\text { statutionis, atque } \\
\text { jmhlicationis, infra ipsius } \\
\text { anni unius integri } \\
\text { revolutionem; Quieunqtie } \\
\text { statulioni praenarratae } \\
\text { contradixcrint, vei etiam ali: } \\
\text { qui postea causar, et liti ipsi } \\
\text { sese innerere, et inunisccre } \\
\text { voluerint, sive Gint filiae } \\
\text { illius defuncti in domo } \\
\text { paterna relictae: sive fratres } \\
\text { generationales: sive autem } \\
\text { caeteri foeminei sexus } \\
\text { homines, jura sua } \\
\text { producendo, coram Judice } \\
\text { suo ad id specialitcr, et ex } \\
\text { presse deputato; Nunc } \\
\text { videlicet coram } \\
\text { Domino .ludicc curite } \\
\text { Regiae(nam alias coram } \\
\text { Domino Palatino } \\
\text { consueveranl) sive } \\
\text { celli, cui Alajestas Regia, bona } \\
\text { praenotata contulerit. } \\
\text { relinquuntur possidenda. } \\
\text { non, ad se, et ad jus eorum } \\
\text { pertinere tenentur } \\
\text { comprobare: Aliter autem }\end{array}$ & $\begin{array}{l}\text { semine defecit, tempore } \\
\text { videlicet legitimae statutionis } \\
\text { eorum, praesentibus vicinis, } \\
\text { et commetaneis, fieri debet, } \\
\text { et a die ejuscemodi } \\
\text { statutionis, atque } \\
\text { publicationis, infra ipsius } \\
\text { anni unius integri } \\
\text { revolutionem; Quicunque } \\
\text { statutioni praenarratae } \\
\text { contradixerint, vel etiam ali: } \\
\text { qui postea causae, et liti ipsi } \\
\text { sese ingerere, et immiscere } \\
\text { voluerint, sive sint filiae } \\
\text { illius defuncti in domo } \\
\text { paterna relictae: sive fratres } \\
\text { generationales: sive autem } \\
\text { caeteri foeminei sexus } \\
\text { homines, jura sua } \\
\text { producendo, coram Judice } \\
\text { suo ad id specialiter, et } \\
\text { expresse deputato; Nunc } \\
\text { videlicet coram Domino } \\
\text { Judice curiae Regiae (nam } \\
\text { alias coram Domino } \\
\text { Palatino consueverant) sive } \\
\text { celebrentur octavae, sive } \\
\text { non, ad se, et ad jus eorum } \\
\text { pertinere tenentur } \\
\text { comprobare: Aliter autem } \\
\text { juri Regio et per consequens } \\
\text { illi, cui Majestas Regia, bona } \\
\text { praenotata contulerit, } \\
\text { relinquuntur possidenda. }\end{array}$ & $\begin{array}{l}\text { uicinis, \& commetaneis fieri debet / \& a die eiufcemodi } \\
\text { ftatutionis, } \\
\text { atque publicationis infra ipfius anni unius integri } \\
\text { reuolutionem. Qui } \\
\text { cunque ftatutioni praenarratae contradixerint, uel etiam } \\
\text { alii / qui poftea } \\
\text { caufae, \& liti ipfi / fefe ingerere, \& immifcere uoluerint / } \\
\text { fiue fint fi= } \\
\text { liae illius defuncti / in domo paterna relictae: fiue fratres } \\
\text { generatio= } \\
\text { nales: fiue autem caeteri foeminei fexus homines / iura } \\
\text { fua producen= } \\
\text { do / coram iudice fuo / ad id fpecialiter / \& expreffe } \\
\text { deputato. Nunc } \\
\text { uidelicet coram domino iudice curiae regiae (nam alias } \\
\text { coram do= } \\
\text { mino Palatino confueuerant) fiue celebrentur octauae, } \\
\text { fiue non, } \\
\text { ad fe, \& ad ius eorum pertinere tenentur comprobare. } \\
\text { Aliter autem iuri } \\
\text { regio, \& per confequens illi / cui maieftas regia bona } \\
\text { praenotata } \\
\text { contulerit relinquuntur poffidenda. }\end{array}$ \\
\hline
\end{tabular}

\section{Werbőczy und die Ressourcen des Internet}

Bis hierher ging es nur um die Digitalisierung des lateinischen Textes des Opus Tripartitum von 1517/1822 und dessen deutscher Übersetzung von 1599. Schon allein das wäre ohne das Internet und seine Möglichkeiten nicht realisierbar gewesen.

Aber was kann im heutigen Zeitalter der Informationsvernetzung darüber hinaus als Zugang zu diesem Autor und seinem Text angeboten werden? Eine einfache Suche nach dem Namen Werböcsy über Google ergab etwa 10.500 Ergebnisse [25.05.2020 14:21], wobei auch Schreibformen mit „„” angezeigt wurden. Die Suchalgorithmen von Google mögen einigen wenigen Fachleuten bekannt sein - mir sicherlich nicht. Aber es gibt Filterungsmöglichkeiten, etwa indem man die angezeigten Ergebnisse auf solche in deutscher Sprache beschränkt, dies wären 
dann in diesem Fall etwa 110 Ergebnisse. Ich darf das Resultat vorwegnehmen: Die Durchsicht dieses Suchergebnisses ergab wenig Brauchbares, manche Hinweise auf Literatur mit zumindest der Nennung von Werböczy, selten aber wirklich weiterführendes Material. Dazu gehört (an 29. Stelle des Ergebnisses und als einziger Verweis auf das Repertorium) der Hinweis auf einen Abschnitt über Werböcayy bei Roman Schnur, ${ }^{32}$ den ich durch das Entgegenkommen des Verlages digitalisieren durfte.

Letztlich bleibt als beste Recherchemöglichkeit der Rückgriff auf die eigenen Kenntnisse und die Bücher, aus denen sie im Laufe der Zeit gewonnen wurden. Es sei mir nachgesehen, dass auch ich dies - trotz meiner unbestreitbaren Internetaffinität - mit einer gewissen Befriedigung vermerke. Denn auch Projekte wie DRQEdit und das Repertorium dienen letztlich dem Zweck, zusammengehörige Informationen in einem Portal zusammenzustellen, so dass die Zufallsergebnisse einer Google-Suche ersetzt oder ergänzt werden können durch ein themenzentriertes und fachwissenschaftlich basiertes Wissensangebot.

Und wenn ich oben von einem „Handwerkszeug“ gesprochen habe, so ist dies manchmal cum grano salis zu verstehen. Ein Beispiel möge dies verdeutlichen. Im ungarischen Recht und damit auch bei Werbö́çy spielt das Donationalsystem eine große Rolle, das dem deutschen Rechtshistoriker unbekannt ist. Wollte ich zumindest einen ersten Zugang zu solchen Besonderheiten über die entsprechende Sachliteratur zur Verfügung stellen, so musste ich danach erst suchen. Und zwar möglichst im Bereich der deutschsprachigen Sekundärliteratur, wo auch die deutsche Übersetzung (Berlin 1904) ${ }^{33}$ einer ungarischen Verfassungs- und Rechtsgeschichte von Akos von Timon zu finden war. Der bloße Hinweis auf dieses Werk schien mir allerdings nicht zu genügen. Und so zerlegte ich die bei der Bayerischen Staatsbibliothek herunterladbare PDF-Datei in Einzelseiten, die einerseits direkt adressierbar sind und die andererseits die Grundlage für die Digitalisierung des Inhaltsverzeichnisses mit einer Verlinkung zu den jeweiligen Seiten ermöglichte. Mit der Digitalisierung des Inhaltsverzeichnisse ${ }^{34}$ wäre mein Ziel erreicht gewesen. Im dritten Kapitel des dritten Buches findet sich: §. 6. Die Grundprincipien des Donationalsystems.

Allerdings: Da ich in diesem Projekt auch Seitenwegen zu folgen bereit bin, habe ich dann damit begonnen, Teile des Werkes von Ákos von Timon zu digitalisieren. Denn in diesem Werk werden Begriffe und Institutionen abgehandelt, die zumindest für mich schon bei der Lektüre des Opus Tripartitum teilweise erklärungsbedürftig waren: Donation, Fassion, Aviticität, Oktaue, beglaubigter Ort, Statution, Quartalitium etc. Hier zeigen sich allerdings auch die Grenzen einer nur der eigenen Willkür bzw. dem eigenen Interesse verpflichteten Digitalisierungsstrategie: Einer Idealvorstellung folgend, sollte jedes Quellenzitat bzw. jeder Quellenhinweis über eine Verlinkung zur jeweiligen Quelle und ihrem Digitalisat führen. Ich habe dies testweise bei einem wichtigen Quellenwerk der ungarischen Geschichte ausprobiert: György Fejér, Codex Diplomaticus Hungariae ecclesiasticus ac civilis 1 (1829) - 12, 2 (1862). Das Gesamtwerk ist von der Bayerischen Staatsbibliothek digitalisiert und in deren digitalem OPAC erfasst worden. Es lag nahe, in meinem Repertorium eine verlinkte Liste der Bände anzubieten (Tabelle). ${ }^{35}$ Dies hatte aber einen weiteren

\footnotetext{
32 SCHNUR, Roman: Die Rolle der Juristen bei der Entstehung des modernen Staates. Berlin 1986.

33 http://repertorium.at/img/AvTimon_UngVerfgesch_1904/index.html.

34 http://repertorium.at/sl/AvTimon_Inhalt.html.

35 http://repertorium.at/territorien.html\#ungarn.
} 
Grund in der Absicht, zumindest manche der Zitate in Timons Werk verlinken zu können. Es würde zu weit führen, hier in Einzelheiten zu gehen, aber im Endeffekt kann ich bei der Digitalisierung des Timonschen Werkes nun bei jeder Fußnote, die den Codex Diplomaticus Hungaricus zitiert, vermittelst einer von mir festgelegten Kurzform (Entität: \&cdiplhung_fejer_1;) zumindest einen Link auf den jeweiligen Band einfügen. Der Benutzer / die Benutzerin wird dadurch mit dem Digitalisat der BSB verbunden und kann im dortigen Menü die ihn / sie interessierende Seite ansteuern. Dies gilt inzwischen (es handelt sich schließlich um ein work in progress) auch etwa von Gustav Wenze ${ }^{6}$ oder Kovachich. ${ }^{37}$ Noch komfortabler wäre es gewesen, jedes Zitat unmittelbar mit der zitierten Seite zu verbinden, aber das wäre nun wirklich zu zeitaufwendig geworden.

Was das Repertorium zu Ungarn und Werböcsy anbietet, ist hier zu sehen, allerdings ohne die Bandliste des Codex Diplomaticus, die auch etwas umfänglich geworden ist:

\section{Quellen}

Stefan von Werbőczy, Opus Tripartitum [Wien 1517] Digitalisat der Österreichischen Nationalbibliothek ${ }^{38}$

Paralleldarstellung Seite / Transkription [in Arbeit 10.05.2020] ${ }^{39}$

Stefan von Werbőczy, Opus Tripartitum ... deutsche Übersetzung [Wien 1599] Digitalisat und Titelaufnahme in DRQEdit ${ }^{40}$

Stefan von Werbőczy, Opus Tripartitum ... deutsche Übersetzung [Wien 1599] Transkription Heino Speer 2019/20 [der Text mit originalem Zeilenfall wird später bereitgestellt] ${ }^{41}$

Corpus Juris Hungarici, Werbőczy, István, 1458-1541 (Budae 1822). Digitalisat ÖNB ${ }^{42}$

Corpus Juris Hungarici, Tripartitum (Milleniumsausgabe 1897) ${ }^{43}$

Werbőczy, István, Opus Tripartitum Juris Consuetudinarii (lat. 1517/1822): Decretvm oder Tripartitvm Opvs (dt. 1599): ParalellausgabeTranskription und digitale Formatierung Heino Speer (neu in der Arbeit). ${ }^{44}$

Konkordanz:

Ausgabe 1517 (Faksimile)

1599 (deutsche Übersetzung: Faksimile und Volltext)

1822 (Corpus Juris Hungarici: Faksimile)

1897 (Corpus Juris Hungarici, Milleniumsausgabe: Faksimile) ${ }^{45}$

\section{Sekundärliteratur}

Timon, Ákos von: Ungarische Verfassungs- und Rechtsgeschichte (Berlin 1904) [BsB-Digitalisat als herunterladbare PDF-Datei] ${ }^{46}$

Timon, Ákos von: Ungarische Verfassungs- und Rechtsgeschichte (Berlin 1904) [BsB -Digitalisat, in einzelne JPEGDateien aufgeteilt, die seitengenau verlinkt werden können. Repertoriumsbearbeitung $]^{47}$

Mit den Seiten verlinktes Inhaltsverzeichnis von Timon, Ákos von: Ungarische Verfassungs- und Rechtsgeschichte

\footnotetext{
${ }^{36}$ https://portal.dnb.de/opac.htm?method $=$ simpleSearch\&cqlMode $=$ true\&query $=$ nid\%3D133081095.

${ }^{37}$ https://portal.dnb.de/opac.htm?method $=$ simpleSearch\&cqlMode $=$ true\&query $=$ nid $\% 3 D 1018228063$

38 http://digital.onb.ac.at/OnbViewer/viewer.faces?doc=ABO_\%2BZ197419403.

39 http://repertorium.at/qu/1517_opus_tripartitum_transkription.html.

40 https://drw-www.adw.uni-heidelberg.de/drqedit-cgi/zeige?index=siglen\&term=tripartitum\%201599\%22.

${ }^{41}$ https://digital.onb.ac.at/OnbViewer/viewer.faces?doc=ABO_\%2BZ15940550X.

42 https://digital.onb.ac.at/OnbViewer/viewer.faces?doc=ABO_\%2BZ15940550X.

${ }^{43}$ https://adtplus.arcanum.hu/hu/view/Eltekonyvek_BE_4107_02/?pg=75\&layout=s.

44 http://repertorium.at/qu/opus_tripartitum_lat-dt_tabelle.htm.

45 http://repertorium.at/qu/opus_tripartitum_konkordanz.html.

46 https://opacplus.bsb-muenchen.de/Vta2/bsb00090086/bsb:BV014062532?page=1.

47 http://repertorium.at/img/AvTimon_UngVerfgesch_1904/index.html.
} 
(Berlin 1904) [Repertoriumsbearbeitung] ${ }^{48}$

Timon, Ákos von: Ungarische Verfassungs- und Rechtsgeschichte (Berlin 1904) S. 642-646 [Zu Stefan von Werbőczy] $]^{49}$

Belá Reitzer, Heimfall und Erbfolge. Studie aus dem Kreise des ungarischen Privatrechts mit Rücksicht auf den ungarischen Allg. Bürgerl. Gesetzentwurf (Budapest 1906) [Transkription Speer 2019] ${ }^{50}$

Schnur, Roman, Die Rolle der Juristen bei der Entstehung des modernen Staates (Berlin 1986) Digitalisiert: Ungarische Juristen am Ausgang des Mittelalters S. 66-70 über Stefan von (István) Werbőczy ${ }^{51}$

\section{Hilfsmittel}

Eötvös, Jgnacz baro, Extractus synoptico-encyclopaedicus legum in corpore juris Hungarici contentarum [Budae: Typis Universitatis; 1829] ${ }^{52}$

Stephanus Dudits, Nomenclator operis tripartiti juris consuetudinarii regni Hungariae a Stephano de Verbeucz [Pest: 1829: Google-Digitalisat ${ }^{53}$

Thewrewk de Ponor, Jozsef, Werbőczy Istvan Diák Műszavai régi magyarításokkal. [Stephan Werbőczy's lateinische Kunstausdrücke mit altungarischer Übersetzung](Pozsony: Schmid, 1844) 54

Rechtsungarisch: deutsch-ungarisches und ungarisch-deutsches Rechtswörterbuch für jedermann / von Gerhard Köbler; unter Mitarb. von Marianna Schenk und Kasia Sobiecka. [Giessen: Arbeiten-zur-Rechts-undSprachwissenschaft Verlag, 2004] ${ }^{55}$

Verzeichnis ungarischer Wörterbücher für historische Forschungen [Quelle: Österreichische Gesellschaft für Familien- und regionalgeschichtliche Forschung (ÖFR) $]^{56}$

In obiger Auflistung der auf Ungarn bezüglichen Digitalisate im Repertorium gibt es den Punkt: „Konkordanz”. Und darin sollte der Werbőczysche Text auch mit der Milleniumsausgabe von 1897 verlinkt werden. Dies gibt mir den Anlass, auf ein grundsätzliches Problem im Umgang mit digitalen Ressourcen einzugehen.

Es hatte längere Zeit gedauert, bis ich ein Digitalisat der Milleniumsausgabe des Corpus Juris Hungarici von 1897 gefunden hatte. Es war auf der Seite einer ungarischen Institution [Arcanum. Digitális Tudománytár] zu finden, deren Status ich wegen meiner fehlenden Kenntnisse des Ungarischen nicht abschätzen konnte. Mir genügte jedenfalls zunächst, dass ich diese Edition in digitaler Form verfügbar hatte. Ich machte mich, nachdem ich durch eine Registration bei dieser Institution den Zugang erwirkt hatte, daran, jede Seite mit den anderen zu verlinken. Als diese Arbeit erledigt war und ich aus der fertigen Konkordanz einen Link aktivieren wollte, bekam ich allerdings kein Seitenfaksimile zu sehen, sondern folgenden Text: „Dokumentum megtekintése elofizetést igényel Bejelentkezés | További információk Ezekben az intézményekben ingyen bozzáférhet a tartalomboz: Keressen és böngésszen ingyen”.

Es bedurfte einer Anfrage an eine ungarische Wissenschaftlerin, bis ich diese Meldung verstehen konnte. Sie besagte schlichtweg, dass es sich um ein kostenpflichtiges Angebot handelte.

\footnotetext{
48 http://repertorium.at/sl/AvTimon_Inhalt.html.

49 http://repertorium.at/sl/timon_ungarische_verfassungsgeschichte_1904.html.

50 http://repertorium.at/sl/reitzer_heimfall_1906.html.

51 http://repertorium.at/sl/schnur_werboeczy.html.

52 https://digital.onb.ac.at/OnbViewer/viewer.faces?doc=ABO_\%2BZ137185805.

53 https://books.google.at/books?id=ScNIAAAAcAAJ.

54 https://digital.onb.ac.at/OnbViewer/viewer.faces?doc=ABO_\%2BZ16762090X.

55 http://www.koeblergerhard.de/Rechtsungarisch/deutsch-ungarisch1.pdf.

56 https://wiki.oefr.at/W\%C3\%B6rterb\%C3\%BCcher_-_Lexika\#Ungarisch.
} 
Genau das ist das Problem, dessentwegen die Open-Access-Bewegung ins Leben gerufen wurde. Gemeinfreie, zur nationalen und übernationalen Geschichte gehörende Drucke werden digitalisiert, meist auf Grund der Förderung durch öffentliche Einrichtungen. Und dann wird der Zugang kostenpflichtig gemacht, so dass die wissenschaftliche Öffentlichkeit nur unter sehr erschwerten Bedingungen darauf zugreifen kann.

Ein Vorhaben wie das Repertorium, das der Demokratisierung von Wissen dienen soll, setzt aber eine freie Verfügbarkeit der digitalen Ressourcen voraus. Alles andere wäre exklusiv im Wortsinne!

Ich musste daher die Links auf die Millenniumsausgabe von 1897 - zumindest vorläufig deaktivieren. Und nun muss ich warten, bis die gegenwärtigen Einschränkungen durch das CoronaVirus ein Ende gefunden haben und die Bibliotheken, die ich wegen einer weiteren Digitalisierung angeschrieben habe, antworten können. Jedenfalls wäre meine Antwort auf eine solche Restriktion, ein anderes Exemplar dieser Edition woanders digitalisieren zu lassen, und dann auch - aus Prinzip - auf meine eigenen Kosten. Und wenn dies erfolgt ist, nehme ich auch gerne die Mühen einer erneuten Verlinkung auf mich. Und am Rande sei bemerkt: Alle meine Publikationen sind frei und unentgeltlich im Internet verfügbar: OPAC der UB Heidelberg ${ }^{57}$ (Suchbegriff: Heino Speer).

\section{Fazit}

Es bleiben aber noch zwei Fragen übrig: Warum nimmt man diese Mühe auf sich und wieweit werden solche Angebote im Internet überhaupt wahrgenommen. Man könnte dies auch als Kosten-Nutzen-Analyse bezeichnen.

Meine Motivation ist mehrschichtig, innere und äußere Momenten spielen hier zusammen. Rein subjektiv: Ich mag diese alten Drucke, auch wenn von deren haptischem und optischem Reiz in der Bildschirmdarstellung wenig übrigbleibt. Und ich finde es faszinierend, in einer Internetplattform Dinge zusammenbringen zu können, die als reale Objekte niemals zusammenkämen. In DRQEdit gibt es Druckwerke, die nur ein- oder zweimal auf der Welt vorhanden sind. Kaum jemand hätte ohne das Internet und ein solches Portal die Gelegenheit, sich damit zu beschäftigen. Ein weiterer Grund ist vielleicht ganz banal: Ich kann mir das leisten. Ich bin im Ruhestand, habe einen gewissen Wissensfundus angesammelt, den ich jetzt verwerten kann. Und ich kann es mir deswegen leisten, weil ich keine Laufbahn mit meiner Arbeit vorbereiten und begründen muss. „Otium cum dignitate” (Cicero), das ist eine der schönsten Grundlagen wissenschaftlichen Arbeitens im Alter.

Aber wieweit wird nun ein solches Webportal im Internet gewürdigt? Ich glaube, diese Frage ist im Internet nicht mehr von Belang. Man mag das bedauern, aber das Internet ist geprägt von einer Konsummentalität, die selten zu der Hinterfragung oder gar Würdigung eines konsumierten Angebots durchdringt. Ich habe beispielsweise nur einmal eine Reaktion auf mein Repertorium bekommen. Entscheidender ist wohl, wie oft eine solche Seite mit ihren Unterangeboten aufgerufen wird. Google bietet eine solche Auswertung inzwischen an und danach liegen die Zugriffszahlen je Monat über 1.000. Und es zeigt sich, dass auch ein historisches Angebot für aktuelle Fragen genutzt wird. So betraf ein Aufruf das Österreichische Allgemeine Bürgerliche

\footnotetext{
${ }^{57}$ https://www.ub.uni-heidelberg.de/helios/kataloge/heidi.html.
} 
Gesetzbuch von 1811, das auch mit dem aktuell geltenden Gesetzestext verlinkt ist, und das Stichwort „Seuche” ... Nach dem Auftreten des Coronavirus, selbstverständlich.

Wenn es hier gelungen wäre, ein wenig darüber aufzuklären, welchen Hintergrund solche Angebote haben können und dass sie immer schlichtweg auf viel Arbeit beruhen, so hätte sich dies ja gelohnt. Und für Rückfragen wäre immer dankbar Heino Speer per Email heino.speer@repertorium.at. 